\title{
Adapting the CSE Program at ETH Zurich to the Bologna Process
}

\author{
Rolf Jeltsch and Kaspar Nipp \\ Seminar for Applied Mathematics, ETH Zurich, CH-8092 Zurich, Switzerland \\ jeltsch@math.ethz.ch, nipp@math.ethz.ch
}

\begin{abstract}
In 1997 a new interdisciplinary Diploma program in Computational Science and Engineering (CSE) was started at ETH Zurich. We report on the changes of the curriculum due to the Bologna Declaration of June 19, 1999 by the European Ministers of Education. The new Bachelor program in CSE at ETH Zurich started in October 2003 and the Master program in CSE will follow in October 2005. We will describe both programs in some detail as well as the design principles. We will also discuss the boundary conditions imposed by ETH and we will give some preliminary experiences.
\end{abstract}

\section{Introduction}

In the 2002 International Conference on Computational Science, ICCS, held in Amsterdam, April 21 - 24, we have reported on the CSE Program at ETH, see [1]. This program was started in 1997 and was leading to a Diploma degree in CSE. In brief, students could join the CSE curriculum after 4 semesters of studies in a subject related to CSE (e.g., engineering, computer science, mathematics, physics, science). The CSE curriculum consisted of additional 4 semesters of studies followed by a diploma thesis to be completed in 4 months. The details of the curriculum are given in [1]. We report on the changes of the curriculum due to the Bologna Declaration of June 19, 1999 by the European Ministers of Education. ETH Zurich wanted to be among the first to change the diploma curricula into Bachelor and Master programs. The new Bachelor program started in the fall of 2003 with about 15 students and the Master program will be started in the fall of 2005. We will describe here the basic design principles of the new programs. For the design of the new Bachelor/Master curricula in CSE we still had the same understanding of the new discipline Computational Science and Engineering (CSE) and what we feel a student in this subject should learn in the program.

\section{The Bachelor and Master Curriculum in CSE at ETH}

\subsection{Design Principles and Boundary Conditions}

The transformation of the previous diploma studies into the new two stages program had to follow the objectives we had intended for the diploma studies and some boundary conditions imposed on us by the ETH system. 


\section{a) Aim of Curriculum}

When designing a new curriculum one should think of what students should know and which abilities they should have acquired when successfully finishing the program. Our principles for the Bachelor and Master program are basically the same as for the Diploma curriculum in CSE, see [1].

The major objectives and design principles are:

- The emphasis is on Science and Engineering and not just on computing for the sake of computing, i.e., we want that the students know what they are computing.

- Computing has to be an essential tool in any field taught.

- Students should make an in-depth study in at least one or maybe two fields.

- Students should obtain a broad view and should have knowledge of many applications.

- Students should acquire the ability to work in a team.

- Students should have the ability to work together with people from different backgrounds (e.g., they must be able to talk to scientists and/or engineers who know the application area and they must be able to talk to mathematicians and computer scientists since they cannot solve all problems themselves).

- Students should have the ability to enter quickly into an ongoing research project of a team, make a contribution and transmit it successfully to the team.

- Students should acquire communication abilities.

- The overall study time for the Bachelor and Master programs should not be longer than for other students at ETH Zurich, i.e., 4 years and an additional 4 months for the diploma thesis.

\section{b) ETH Boundary Conditions}

Let us first discuss the two major new boundary conditions.

- With the change from the diploma curriculum to the two stages Bachelor/Master program, the major exam after 4 semesters has been abandoned. In our diploma program this used to be the natural point to motivate students from different studies to join the CSE program. This had the advantage to still have two years to educate them in the direction of CSE. It did have, however, the disadvantage that students entered the program with rather diverse backgrounds leading to an individually designed program for each student to fill the gaps.

- ETH kept the first major exam to be done by the students two semesters after entering ETH. This exam is now called basic exam and is much the same in most Bachelor programs.

Therefore, it was natural to start the CSE training directly after the first year of education. Considering the whole period including the master program this gave us an additional two semesters where the selection of courses could 
be tailored to the education in CSE. In addition, since students have no longer strongly diverse backgrounds the filling of the gaps could be abolished.

There is a third dramatic boundary condition, namely, that any two stages Bachelor/Master program should fit the old Diploma program in length. In classical diploma studies one considered the first two years as the basic education terminated by the above mentioned major exam after 4 semesters. Then the second 4 semesters were used to train the students in advanced topics, i.e., in the CSE core courses and in their field of specialization. With the two stages program the Bachelor exam just cuts the time interval for the advanced education exactly in the middle. This meant that we had to make sure that the advanced courses already started in the Bachelor curriculum. For this reason we have designed the Bachelor/Master program as a whole. Concerning the core courses, students have to take all of them during their Bachelor/Master studies. Concerning the Fields of Specialization, we have introduced a minor field in the Bachelor program and a major field in the Master program. Clearly this combined design of the Bachelor/Master program could prolong the Master studies for students from outside. Another problem turned out to be that for mobility reasons students should finish their Bachelor degree by the end of the summer semester and not only in the fall. Hence, the Bachelor thesis should preferably be done during the final semester of the Bachelor studies. This thesis is in the range of a term paper which means that it is about 160 hours of work. Two positive changes at ETH have improved the curriculum in CSE. One is the increase of teaching staff so that we can now offer more courses which are geared directly to this group of students. The other is that we could introduce new fields of specialization, namely, Computational Astrophysics and Computational Chemistry and Biology. The latter replaces Computational Chemistry. In addition, we have introduced a new core course Computational Statistics.

\subsection{Curriculum}

\section{a) The Bachelor Curriculum in CSE}

The CSE Bachelor curriculum consists of two years of studies (4 semesters, second and third year) and is based on knowledge acquired in a first year of basic studies at ETH Zurich or elsewhere. The basic exam after the first year is counted for 60 credit points (ECTS). In the following two years the students have to get 120 ECTS, half of them in mandatory Basic Courses, the other half mainly in eligible Core Courses, Fields of Specialization, Elective Courses and with a Bachelor Thesis, respectively.

The Basic Courses provide basic knowledge in Mathematics, Computer Science and Science and Engineering. They are mandatory. The corresponding examinations are combined in 5 blocks supposed to be taken after the third, fourth and fifth semester, respectively. The list of basic courses is following:

- Analysis III

- Complex Analysis 
- Data Bases

- Programming Techniques for CSE

- Numerical Mathematics for CSE

- Computer Networks

- Parallel Numerical Computing

- Optimization Techniques

- Quantum Mechanics

- Stochastics

- Statistical Physics

- Chemistry for CSE

- Physics I and Physics II

- Fluid Dynamics for CSE

The Core Courses are concerned with computational mathematical methods and with more advanced topics in computer science. Students have to take 2 of them in the third year and pass a combined exam. The list of core courses is following:

- Theory and Numerical Techniques of Differential Equations

- Computational Statistics

- Software Engineering

- Visualization / Graphics

The Fields of Specialization provide a profound knowledge in application areas with an emphasis on computational aspects. In the third year, students have to take 2 courses in a chosen field of specialization and pass the exams. The list of specialization fields is following:

- Astrophysics

- Physics of the Atmosphere

- Chemistry and Biology

- Fluid Dynamics

- Control Theory

- Robotics

- Theoretical Physics

The Elective Courses are complementary to the Fields of Specialization and to the Core Courses and should have an emphasis on computational aspects. Students in the third year have to take 2 courses out of a large list and pass the exams. In their third year, students have to attend twice the CSE Case Studies Seminar where each semester invited speakers from ETH, from other universities as well as from industry give a 2x45 minutes talk on an applied topic. Beside of attending the scientific talks students are asked to give short presentations (10 minutes) on a published paper out of a list (containing articles from, e.g., Nature, Science, Scientific American, etc.). In the case studies ETCS are obtained on a passed/failed basis. The Bachelor Thesis concludes the curriculum. It should deepen the knowledge in a specialization area, provide a 
first contact with applications and should enable students to learn to approach applied problems computationally. It should also enable them to work within a scientific group. The bachelor thesis is typically written in a topic of a core course or a field of specialization. It requires about 160 hours of work and ECTS are obtained for an accepted paper.

Table 1. The CSE Bachelor Curriculum

\begin{tabular}{l|lc} 
& Year & ETCS (at least) \\
\hline Basic Studies & Semester 1/2 & $\mathbf{6 0}$ \\
\hline \multirow{5}{*}{ Bachelor CSE } & Semester 3/4 & $\mathbf{6 5}$ \\
& Basic Courses & 65 \\
& Semester 5/6 & $\mathbf{5 5}$ \\
& Core Courses & 17 \\
& Field of Specialization & 6 \\
& Elective Courses & 6 \\
& Case Studies & 6 \\
& Bachelor Thesis & 8
\end{tabular}

After having successfully finished their studies students will be given the degree Bachelor of Science ETH in Computational Science and Engineering (BSc ETH CSE). The main objective of the Bachelor curriculum is to prepare the students for the continuation of their studies in high quality Master curricula.

\section{b) The Master Curriculum in CSE}

The CSE Master curriculum at ETH Zurich consists of one year of studies (2 semesters, fourth year) followed by a Master thesis. The Master curriculum is based on the CSE Bachelor curriculum and its objective is to prepare students for a successful professional career in research in industry and/or on a university level. The Master students have to get 90 ECTS mainly in Core Courses, in eligible Fields of Specialization and Elective Courses as described in Subsection a) and with a Term Paper and a Master Thesis, respectively. In the Core Courses students have to take those 2 courses not covered for the Bachelor degree and pass a combined exam. Students have to choose a second Field of Specialization out of the list given in Subsection a) (in addition to the one chosen for the Bachelor degree) and have to take 4 courses within their two fields of specialization and pass the exams. Students have to take 2 Elective Courses and pass the exams. Students have to attend twice the CSE Case Studies Seminar as described in Subsection a) for the Bachelor curriculum. The Term Paper should be computational and application oriented work in a team in order to deepen the knowledge in a specialization area. It should require about 160 hours and ECTS are obtained for an accepted paper. The Master Thesis takes 4 
months and is advised by a professor teaching in a core course or in a field of specialization. It concludes the CSE Master studies and should teach students to work independently on a scientific topic. ECTS are obtained for an accepted thesis. After having successfully finished their studies students will be given the degree Master of Science ETH in Computational Science and Engineering (MSc ETH CSE).

Table 2. The CSE Master Curriculum

\begin{tabular}{l|lc} 
& Year & ETCS (at least) \\
\hline \multirow{4}{*}{ Master CSE } & Semester 7/8 & $\mathbf{6 0}$ \\
& Core Courses & 17 \\
& Field of Specialization & 16 \\
Elective Courses & 6 \\
Case Studies & 6 \\
Term Paper & 8 \\
& Semester 9 & $\mathbf{3 0}$ \\
& Master Thesis (4 months) & 30
\end{tabular}

\section{Conclusions}

As the program has started just a few weeks before the writing of this manuscript we can report only on preliminary experiences. One is that due to the fact that the Bachelor program starts after one year of studies at ETH we have now students which entered ETH after high school with the aim to study CSE. In the fall of 200314 students have entered the program. Again all students are highly motivated. Currently, there are no foreign students. Hence, the teaching is not done in English. It will be no problem, however, to do so if necessary. For more information on the CSE Bachelor/Master curricula see the webpage www.cse.ethz.ch. Reference [2] may be ordered from the Seminar for Applied Mathematics, ETH Zurich, CH-8092 Zurich.

\section{References}

1. Jeltsch, R., Nipp, K.: CSE Program at ETH Zurich: Are we doing the right thing? Eds. P.M.A. Slott, C.J. K. Tan, J.J. Dongarra, A.G. Hoekstra, Proceedings of the Conference: Computational Science - ICCS 2002, Vol III, pp. 863 - 871.

2. CSE Computational Science and Engineering, Annual Report 2002/2003, R. Jeltsch, K. Nipp, W. van Gunsteren, edits., ETH Zurich, 2003. 